\title{
Assembly of carbon tube-in-tube nanostructures
}

\author{
D. S. Su ${ }^{\text {a }}$ Z.P. Zhu ${ }^{\text {a,b }}$, X. Liu ${ }^{\text {a }}$, G. Weinberg ${ }^{\text {a }}$, N. Wang ${ }^{c}$, R. Schlögl ${ }^{\text {a }}$ \\ a Department of Inorganic Chemistry, Fritz Haber Institute of the MPG, Faradayweg 4-6, \\ D-14195 Berlin, Germany \\ ${ }^{\mathrm{b}}$ State Key Laboratory of Coal Conversion, Institute of Coal Chemistry, Chinese Academy of Sciences, \\ 030001 Taiyuan, China \\ ${ }^{c}$ Physics Department, Hong Kong University of Science and Technology, Hong Kong, China
}

PACS: 81.07.De, 81.16.Pr, 81.16.Fg, 81.16.Hc, 81.16.Be

\begin{abstract}
Tube-in-tube carbon nanostructures were prepared by reorganization of graphitic impurity nanoparticles outside or inside of the pristine carbon nanotubes. Graphitic impurity nanoparticles were first disintegrated into small graphene fragments by a chemical oxidation with nitric acid, which also modifies the graphene fragments with carboxyl and hydroxyl groups at their edges. The functionalized graphene fragments were then reintegrated outside or inside of pristine carbon nanotubes to construct into tube-in-tube nanostructures. The combination of oxidatively functionalized graphene units, their solvate in a polar organic medium allowing for dispersive forces to effect supramolecular organization with carbon nanotubes acting as templates and their polycondensation by acid-catalysed esterification followed by pyrolysis of the oxygen functionalities lead to complex nanostructures inaccessible by direct synthesis.
\end{abstract}

\section{INTRODUCTION}

The surface and channel accessibility of carbon nanotubes (CNTs) bestow them with wide applications in catalysis,[1-4] gas sensing,[5-7] and template-based assembly of heterostructures.[8-11] However, the technical application of CNTs is handicapped by the high production costs and by the byproducts in all the high temperature methods developed so far. A rational, organic wet-chemical synthesis of CNTs becomes the dream of chemists and materials scientists during last 10 years. In the present work, as an intermediate approach between chemical vapor deposition and molecular organic synthesis concepts we report about the synthesis of carbon tube-intube[ 12,13] nanostructures by means of disassembly and assembly of graphitic fragments using ordinary carbon nanotubes as templates. We use non-covalent forces to manipulate graphene fragments to supramolecular tubular structures in a wetchemical process rather different from all current high temperature methods for CNT synthesis(for instance, metal particle catalyzed chemical vapor decomposition of hydrocarbon or arc-discharging of graphite). On the one side, this experiment could provide a new way for the purification of carbon nanotube. It also sheds light to the new application of the carbon material because of the delicate structure and high surface area of carbon tube-in-tube nanomaterials that could be a promising material for hydrogen storage and support material for catalysts. 


\section{EXPERIMENTAL}

The procedure for re-assembling carbon tube-in-tube nanostructures consists of two steps: oxidation and esterification. Firstly $0.25 \mathrm{~g}$ of raw CNTs sample (Applied Sci. Ltd., OH) was combined with $25 \mathrm{ml}$ concentrated $\mathrm{HNO}_{3}$ with magnetic stirring and oxidized for $10 \mathrm{~h}$ under refluxing conditions. After filtration, the remaining solid was washed sequentially with deionized water and ethanol and dried at $110^{\circ} \mathrm{C}$ in air for $12 \mathrm{~h}$.

To synthesis the carbon tube-in-tube nanostructures, $50 \mathrm{mg}$ of the oxidized sample was introduced in $50 \mathrm{ml}$ tetrahydrofuran (THF) by ultrasonic dispersal for $10 \mathrm{~min}$ at room temperature. $0.2 \mathrm{ml}$ of concentrated $\mathrm{H}_{2} \mathrm{SO}_{4}$ was added to the resulted suspension and refluxed for $16 \mathrm{~h}$ to allow a full re-integration of the carboxylhydroxyl- modified graphene fragments through an esterification linkage, in which $\mathrm{H}_{2} \mathrm{SO}_{4}$ served as the acid catalyst. After the reaction, the suspension was filtered, washed with ethanol, and dried at $110^{\circ} \mathrm{C}$ in air for $12 \mathrm{~h}$.

\section{RESUTLS AND DISCUSSION}

As shown in the TEM images (Figure1a, 1b), in raw material the carbon nanotubes are openended, have thin wall (about $20 \mathrm{~nm}$ ) and big inside diameter (from 50nm to 200nm). There are many carbon nanoparticles inside and outside of carbon nanotubes as impurities. After the treatment mentioned above, carbon tube-in-tubes nanostructure were found, as revealed by TEM and SEM images (Figure 1c and 1d). The carbon nanotubes and carbon tube-in-tubes are mixed together (Figure 1c). The yield of carbon tube-in-tubes is more than $5 \%$, which is estimated from the TEM micrographs. The range of diameters of inner and outer tubes is between $50 \mathrm{~nm}$ to 200 $\mathrm{nm}$ and the thickness of walls is about 20nm.The wall thickness of few inner tube is thinner than that of outside one. The carbon tube-in-tube nanostructure is wellconstructed, the thickness is homogenous and the tube end is open. Comparing the electron micrographs before and after treatment, it is obvious that the carbon tube-intube nanostructures are formed through the oxidation and esterification processes.

The concentrated nitric acid used oxidizes the graphitic nanoparticles of carbon nanotubes. In this process, the advantages of acid treatment 1) introducing the $\mathrm{NO}_{3}$ into the interlayer spaces through defect-involved "doors" and exfoliating the large particles into small and thin graphene sheets; 2) modifying the edges of the grapheme sheets with carboxyl and hydroxyl groups through oxidation reaction; 3) removing the amorphous carbon nanopartcles and iron catalyst particles. Although a part of the defected carbon nanotubes and nanoparticle is oxidized, the main carbon nanotubes are not influenced in morphology and structure, which is observed in TEM image (Figure 1c). After oxidation and esterification, there is significant change in morphology and composition, Carbonaceous impurity disappears and the new carbon nanotubes form along the pristine carbon nanotubes inside or outside. It is undoubted that the formation of carbon tube-in-tube is consequence of the assembly of the functionalized graphene sheets. 

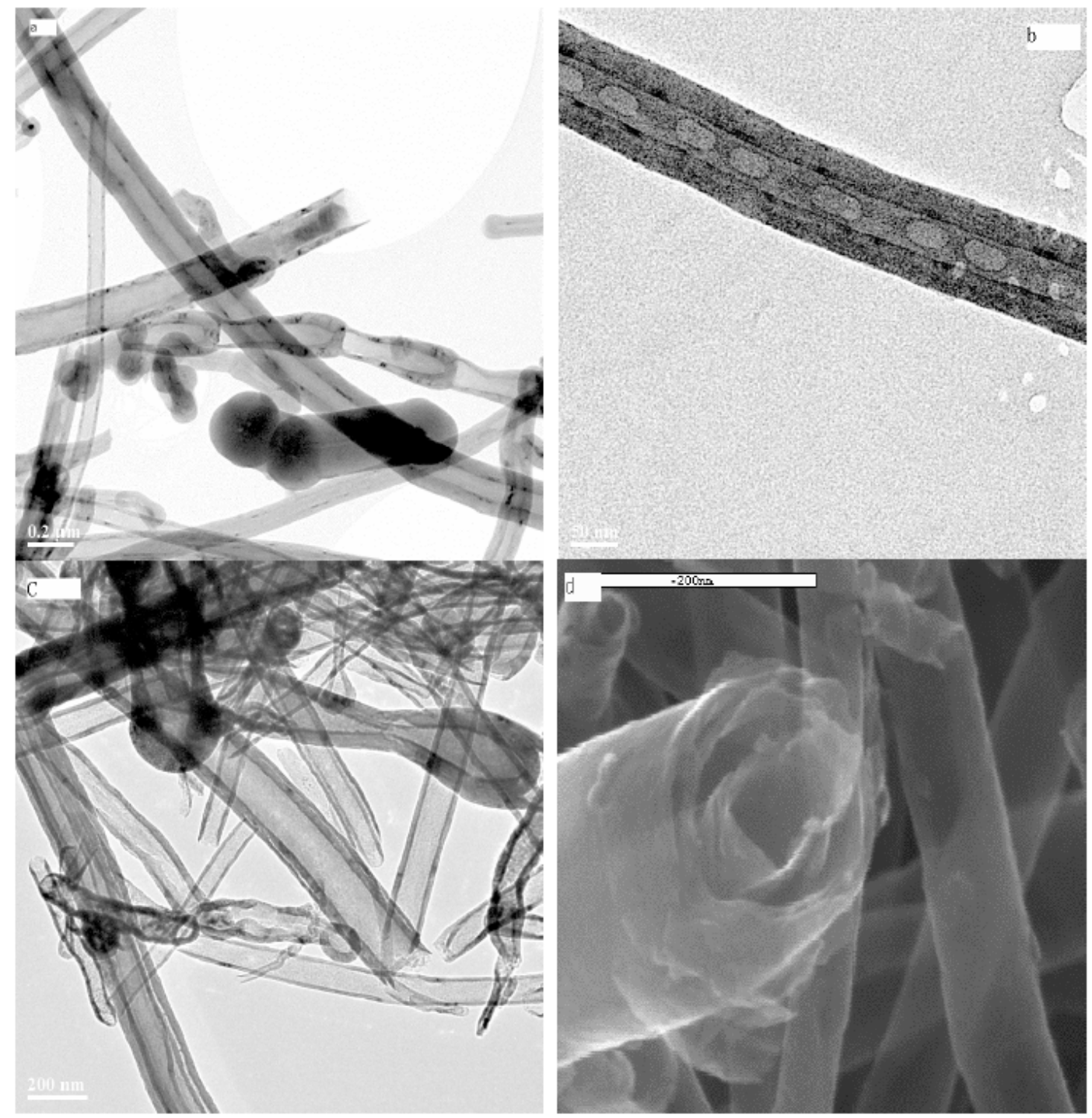

Figure1. a) TEM image of fresh carbon nanotubes with graphite nanoparticles (Applied Sci. Ltd., OH);

b) TEM image of graphitic nanoparticles inside a fresh carbon nanotube;

c) TEM image of carbon tube-in-tube nanostructures and carbon nanotubes;

d) SEM image of carbon tube-in-tube structure.

Another impressive phenomenon is the bamboo-like carbon tube-in-tube nanostructure (Figure 2). A pristine bamboo-like carbon nanotube is shown in Figure 2a. After the treatment, a new bamboo-like tube is formed along with the pristine one , as it is shown in Figure 2b. Obviously the re-assembled carbon nanotube copies twists, nodes and other special shapes of carbon nanotube (inside or outside, see Figure 2b and 2c). This suggests that the pristine carbon nanotubes act as the structure-directing template. It is also clear that the graphene sheets reassemble with the different mechanisms (outside, inside or both in the direction of pristine tubes). Evidently, the graphite particles oxidized and esterified form the carbon tube-in-tube, which morphology is strongly influenced by the matrix carbon nanotube. 


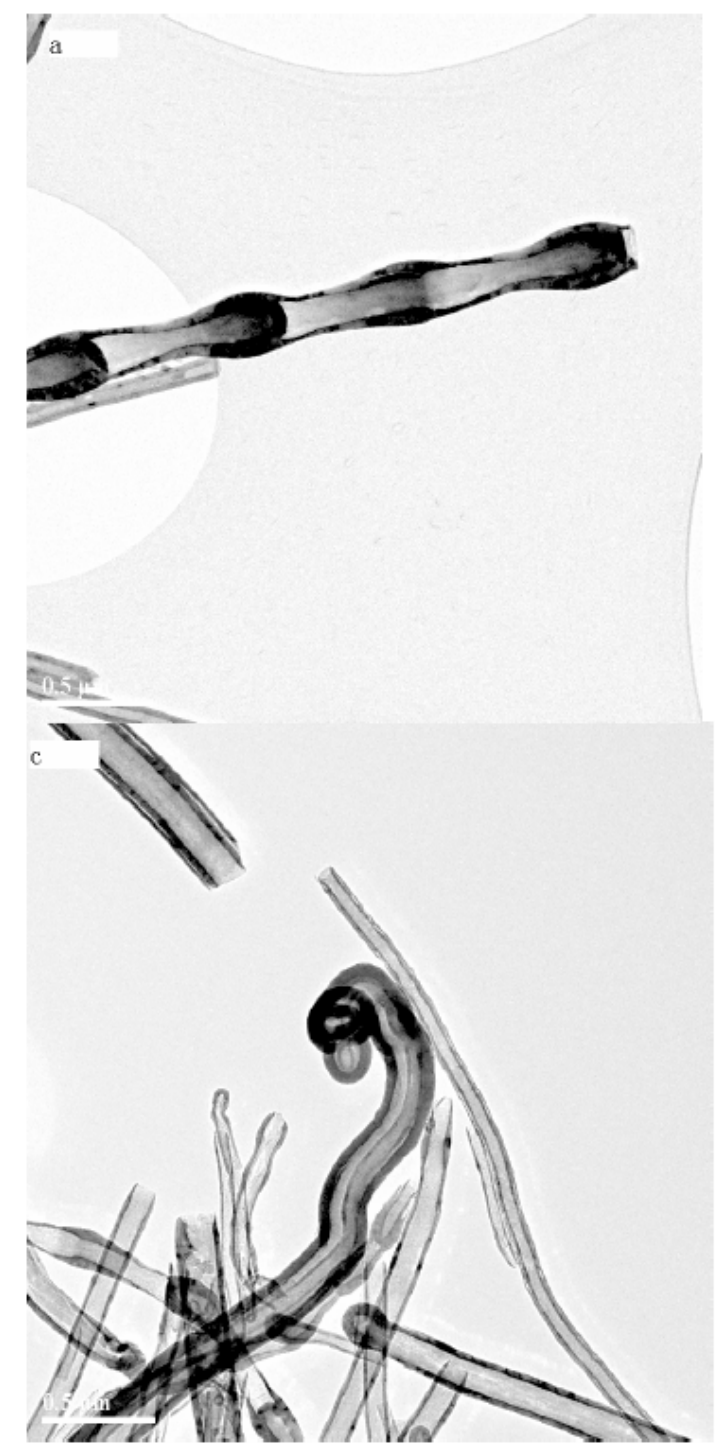

\section{b}

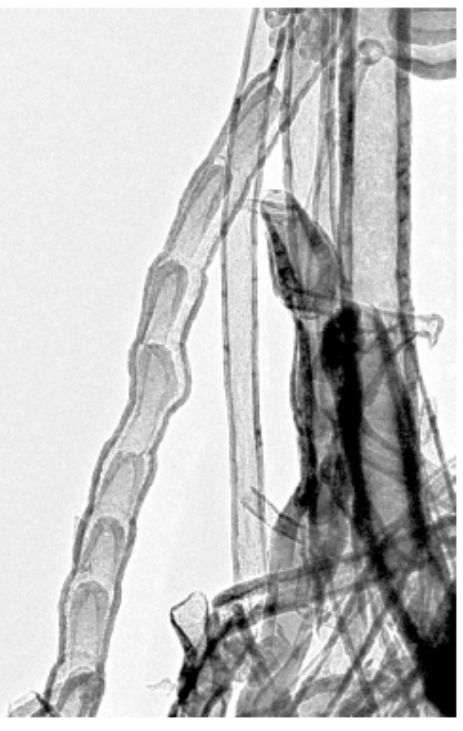

Figure2 a) TEM image of bamboo-like carbon nanotube in raw material;

b) TEM image of bamboolike carbon tube-in-tube;

c) TEM image of carbon tube-in-tube, the new tube forms inside the carbon nanotube.

\section{SUMMARY}

The graphitic impurity among the carbon nanotubes is used as the carbon source for synthesis of new carbon nanotube. After oxidation and the esterification, the graphene sheets re-organize and assemble the carbon tube-in-tube. This strategy would give a light to the new synthesis method of carbon nanotube with low temperature and mild conditions, it also suggests that the new carbon material with the delicate morphology and high surface area would be the good material for hydrogen storage and support material for catalysts. 
AIP Conference Proceedings (2005) Volume 786, pp. 296-300

Electronic Properties of Novel Nanostructures: XIX International

Winterschool/Euroconference on Electronic Properties of Novel Materials

\section{ACKNOWLEDGMENTS}

The work is partially supported by NSFC (59872047) and by the Deutsche Forschungsgemeinschaft (Project SCHL 332), performed in frame of ELCASS.

\section{REFERENCES}

1. Kroto, H.W., Heath, J.R., O’Brien, S.C., Curl, R.F., Smalley, R.E., Nature 318, 162 (1985).

2. Iijima, S., Nature 354, 56 (1991).

3. Liu, J., Dai, H., Hafner, J. H., Colbert, D. T., Smalley, R. E. Nature 385, 780 (1997).

4. Sano, M., Kamino, A., Okamura, J., Shinkai, S. Science 293, 1299 (2001).

5. Martel, R., Shea, H. R., Avouris, Ph. Nature 398, 299 (1999).

6. Falvo, M. R., Clary, G. J., Taylor, R. M., Brooks, F. P., Washburn, Jr. S., Superfine, R. Nature, 389, 582 (1997).

7. Lu, J. P. Phys. Rev. Lett. 79, 1297(1997).

8. Chopra, N. G., Benedict, L. X., Crespi, V. H., Cohen, M. L., Louie, S. G., Zettl, A. Nature 377, 135 (1995).

9. Ugarte, D. Nature 359, 707 (1992).

10 Wang. Z.L., Yin, J.S., Chem. Phys. Lett. 289, 189(1998).

11. Rietmeijer, F.J.M.,, Schultz, P. H., Bunch, Th. E., Chem. Phys. Lett. 374, 464 (2003).

12. Zhu, Z.P., Su, D.S., Weinberg, G., Jentoft, R.E., Schlögl, R., Small 1, 1(2005).

13. Zhu, Z.P., Su, D.S., Weinberg, G., Schlögl, R., Nano Letter 4, 11 (2004). 